\title{
Cálculo das trocas de carbono num agroecossistema de arroz com aplicação de técnicas de preenchimento de falhas
}

\section{Calculation of carbon exchanges in rice agroecossystem with application of Gap-fillin techniques}

\author{
Marcelo Diaz ${ }^{1}$, Debora Regina Roberti ${ }^{1}$
}

${ }^{1}$ Programa de Pós Graduação em Meteorologia, Universidade federal de Santa Maria

\begin{abstract}
Resumo
Estudos em relação às trocas de carbono em ecossistemas agrícolas têm recebido atenção da comunidade científica pois representam um importante papel no ciclo do carbono terrestre. Neste estudado, os fluxos de $\mathrm{CO}_{2}$ obtidos por meio da técnica de covariância dos vórtices sobre uma cultura de arroz irrigado foram utilizados para a estimativa da partição entre respiração e assimilação de carbono pelo agroecossistema. Os dados experimentais foram obtidos em Paraíso do Sul-RS no período de 22/07/2003 a 21/07/2004. O preenchimento de falhas nos dados e o particionamento da serie temporal do fluxo de $\mathrm{CO}_{2}$ experimental foi realizada utilizando uma técnica de regressão não linear que relaciona a respiração do ecossistema com temperatura e a assimilação (produção primaria bruta) com a radiação fotossinteticamente ativa. Ao longo de um ano, a respiração do agroecossistema foi de $1.132 \mathrm{~g} \mathrm{C} \mathrm{m}^{-2}$ e a produção primaria bruta foi de $-1175 \mathrm{~g} \mathrm{C} \mathrm{m}^{-2}$. Desta forma, o agroecossitema apresentou-se como um sumidouro de carbono $43 \mathrm{~g} \mathrm{C}^{-2}$ no ano analisado.
\end{abstract}

Palavras-chave: Covariância dos vórtices, Respiração do ecossistema, Produção Primaria Bruta, Arroz, Técnica de Preenchimento de dados. 


\section{Introdução}

Nos últimos anos, pesquisas relacionadas a trocas de $\mathrm{CO}_{2}$ entre ecossistemas e a atmosfera têm ganhado destaque no meio científico devido ao aumento das emissões antropogênicas de $\mathrm{CO}_{2}$. Estas emissões são causadas pela mudança do uso do solo, queima de combustíveis fosseis, práticas agrícolas, entre outros. Sendo o Brasil um dos principais produtores agrícolas do mundo, caracteriza-se como um dos grandes responsáveis por estas trocas de carbono em função das práticas agrícolas. Desta forma, torna-se de interesse estudar estas práticas a fim de melhor entender seus comportamentos de emissão ou absorção de $\mathrm{CO}_{2}$ (BALDOCCHI et al., 2001).

$\mathrm{O}$ métodomais indicado e utilizado atualmente para estimar as trocas de $\mathrm{CO}_{2}$ entre a atmosférica e o ecossistema é a técnica de covariância dos vórtices (do inglês, eddy covariance) (AUBINET et al., 2000) que relaciona as componente vertical do vento e a concentração do $\mathrm{CO}_{2}$ para obter as trocas líquidas de $\mathrm{CO}_{2}$ no meio quando também se considera o seu armazenamento vertical . Esta técnica pode sofrer falhas, ocasionando lacunas na serie temporal observada. Estas falhas devem ser preenchidas para possibilitar o estudo do balanço anual de $\mathrm{CO}_{2}$ no ecossistema estudado. Para isso, existem inúmeras técnicas, tais como Mean Diurnal Variation, regressões não lineares, Look For UpTables entre outras (MOFFAT et al., 2007).

Modelos matemáticos de regressões não lineares (LASSLOP et al., 2010) que relacionam a temperatura do ar e a radiação solar com as trocas de carbono observadas são, em geral, utilizados para o preenchimento das lacunas nos dados experimentais (troca líquidas de $\mathrm{CO}_{2}$ entre a atmosfera e o ecossistema - NEE), e particionamento do NEE entre a respiração do ecossistema (Re) e a assimilação ou produção primaria bruta (GPP) (definições seguem KIRSCHBAUM et al., 2001). Re e GPP estão fisiologicamente relacionados à temperatura do ambiente e à radiação fotossinteticamente ativa (PAR), respectivamente(ZEIGER et. al., 2009).

Neste estudo, dados experimentais de NEE obtidos sobre um agroecossistema de arroz irrigado no sul do Brasil foram utilizados para a estimativa da suas respiração e GPP. Assim, pode-se estimar se o agroecossitema comportou-se como uma fonte ou sumidouro de $\mathrm{CO}_{2}$ da atmosfera.

\section{Materiais e métodos}

\subsection{Sitio experimental e dados observados}

Nos anos de 2003 e 2004, o Laboratório de Micrometeorologia da UFSM realizou observações micrometeorológicas no município de Paraíso do Sul, no estado do Rio Grande do Sul, numa área de cultivo de arroz irrigado por inundação $\left(29^{\circ} 44^{\prime} 39,6^{\prime \prime} S\right.$; 53 8' 59,8' W, 108 m). A torre instrumentada fazia parte do projeto CT-HIDRO, onde o período de dados utilizados neste trabalho foi de22/07/2003 a 21/07/2004. O cultivar de arroz utilizado para esta safra foi o IRGA 417, o qual foi cultivado no período de 25 de novembro de 2003 até 4 abril de 2004 totalizando 131 dias. O campo foi alagado 30 dias após a semeadura e permaneceu com uma lâmina de água de $5-10 \mathrm{~cm}$ de espessura durante todo o período de cultivo. No restante do período o solo permaneceu em pousio, coberto apenas com uma vegetação rasteira de crescimento espontâneo.

As seguintes medidas de interesse neste trabalho, foram realizadas na área experimental (altura do sensor acima do solo, fabricante, modelo): componentes do vento e temperatura do ar $(10 \mathrm{~m}$, Campbell Scientific, CSAT 3); $\mathrm{H}_{2} \mathrm{O} / \mathrm{CO}_{2}$ analisador de gás e pressão $(10 \mathrm{~m}$, LiCor, LI7500); radiação de onda curta (8 m, Kipp \& Zonen, CMB6); radiação de onda longa (8 m; Kipp \& Zonen, CG); precipitação (10 m; Texas Instruments, CSI Model TB4 Raingage).

\subsection{Calculo do NEE e filtros utilizados}

O fluxo turbulento de $\mathrm{CO}_{2}$ foi obtido através das medidas de alta frequência da velocidade vertical do vento e da concentração de $\mathrm{CO}_{2}$ na atmosfera por meio da técnica de covariância dos vórtices (AUBINET et al., 2000). Foi aplicada a rotação de coordenadas (KAIMAL; FINNIGAN, 1994) para que o sistema coincida com a componente media da direção do vento, assim como a correção em relação às flutuações de densidade do ar, indicadas por Webb et al. (1980). O fluxo de $\mathrm{CO}_{2}$ foi processado em janelas de meia hora. Mesmo o termo de armazenamento de $\mathrm{CO}_{2}$ sendo desprezado (Alberto et al 2009), o fluxo de $\mathrm{CO}_{2}$ calculado representa a troca líquida de $\mathrm{CO}_{2}$ entre a atmosfera e o ecossistema (NEE).

Para o controle de qualidade nos dados de NEE, foram retirados valores que representavam o mau funcionamento do sistema de covariância dos vórtices referentes a perda de energia, falhas mecânicas, problemas no armazenamento dos dados assim como períodos destinados a manutenção do sistema, etc. Apesar deste controle nos dados, ainda podem ocorrer valores espúrios que não representam o fisicamente o NEE do ecossistema. Para tanto, os seguinte filtros foram utilizados:

a) valores de fluxos que não correspondem a real capacidade fenológica da cultura de arroz em relação às condições climáticas presentes nos sítios (NEE $>15 \mu \mathrm{mol}$ $\mathrm{m}^{-2} \mathrm{~s}^{-1}$ e NEE $\left.<-50 \mu \mathrm{mol} \mathrm{m} \mathrm{m}^{-2} \mathrm{~s}^{-1}\right)$.

b) o analisador de gás LI-COR LI7500 executar as mensurações através de um caminho aberto, bem como o anemômetro sônico, os quais permitem que intempéries afetem suas medidas. No caso do LI-COR LI7500, que utiliza o espectro do infravermelho para averiguar a concentração de $\mathrm{CO}_{2}$, a presença de $\mathrm{H}_{2} \mathrm{O}$, que tem 
grande absorção neste espectro, interfere na correta medida experimental, resultando em valores irreais. Sendo assim, é necessário que no período de ocorrência de precipitação, os valores de fluxo sejam descartados (RUPPERT et al., 2006), bem como valores de meia hora seguinte para secagem do instrumento.

c) oscilações causadas pela influencia da umidade, entre outras forçantes, acabam por gerar ruídos (spikes) nas mensurações dos sensores de caminho aberto, para isso, se faz necessário o uso de um filtro que compare os fluxos de cada meia hora () com a media () e o desvio padrão () de uma janela móvel de 200 pontos em torno da meia hora () seguindo as equações adaptadas de Pierre Béziat et al. (2009) :

\section{$f_{i}<f_{m i}-\left(2.5 * f_{s d i}\right)$}

ou

$$
f_{i}>f_{m i}+\left(2.5 * f_{s d i}\right)
$$

Assim, separando os dados em períodos diurnos e noturnos (noturno é definido quando $\mathrm{Rg} \leq 10 \mathrm{~W} \mathrm{~m}^{-2}$ ), os valores de que satisfazem as condições acima são descartados.

d) Valores de NEE onde o u* é pequeno, são removidos por indicar valores de baixa turbulência que violam o regime de eddy covariance. Sobre tais condições de calmaria, a turbulência não é capaz de misturar devidamente o ar abaixo do sensor de tal modo que ocorra a drenagem lateral do fluxo pela advecção (AUBINET et al., 2008) subestimando assim os valores de fluxo. Para isto valores de fluxo com $\mathrm{u}^{*}$ menores que $0,22 \mathrm{~m}$ $\mathrm{s}^{-1}$ foram descartados.

Após o processamento dos dados de NEE utilizando os filtros acima, obteve-se um total de $57,44 \%$ de dados descartados. De acordo com Papale et al. (2006) entorno de 20 a $60 \%$ dos dados podem ser rejeitados pelas técnicas de filtragem.

\subsection{Particionamento de NEE}

No período noturno definido como $\mathrm{Rg} \leq 10 \mathrm{~W} \mathrm{~m}^{-2}$, não temos radiação fotossinteticamente ativa significante, então pode-se definir GPP como sendo zero, logo podemos estabelecer que NEE é igual Re. Sabendo que Re responde a temperatura do ar através de um modelo de Arrhenius (LLOYD; TAYLOR, 1994) é possível determinar NEE para o período noturno:

$$
\mathrm{Re}=r b \exp \left[E_{0}\left(\frac{1}{T_{\text {ref }}-T_{0}}-\frac{1}{T_{a r}-T_{0}}\right)\right]
$$

Sendo $r b$ é a respiração na temperatura de referência $\left(\mu \mathrm{mol} \mathrm{m} \mathrm{s}^{-2} \mathrm{~s}^{-1}\right)$ é $E_{0}$ a energia de ativação, ${ }_{a r}$ é a temperatura do ar, $T_{\text {ref }}=10^{\circ} \mathrm{C}$ e $T_{0}=-46,02^{\circ} \mathrm{C}$ obtidos de Lloyd \& Taylor (1994).

Utilizando a equação (1) para estimar os valores de respiração durante o período diurno, torna-se possível estimar o GPP por meio da relação GPP = NEE - Re, logo podemos estimar a relação entre o GPP com a radiação por meio de uma hipérbole retangular (FALGE et al., 2001).

$$
G P P=\frac{\alpha \beta R g}{\alpha R g+\beta}
$$

onde $\alpha$ é a eficiência com que a luz é utilizada pelo dossel e representa a inclinação inicial da curva de resposta á luz e $\beta$ é a taxa máxima de $\mathrm{CO}_{2}$ absorvida pelo dossel na saturação da luz. Os parâmetros foram óbitos usando uma janela de cinco dias utilizando uma regressão não linear dos mínimos quadrados.

\section{Resultados}

\subsection{Condições Ambientais}

As variáveis meteorológicas durante o período de análise são apresentadas na Figura 1. Durante o período do cultivo do arroz, a temperatura média foi de $23,3^{\circ} \mathrm{C}$ e, ao longo do ano $19,1^{\circ} \mathrm{C}$. A radiação global incidente durante o período de cultivo foi, em média, de $271,7 \mathrm{~W}$ $\mathrm{m}^{-2} \mathrm{e}$ ao longo de todo o período de experimento $200,6 \mathrm{~W}$ $\mathrm{m}^{-2}$. A precipitação acumulada durante o período deste estudo foi de1630 $\mathrm{mm}$, sendo para o período de cultivo do arroz $486 \mathrm{~mm}$. A precipitação foi bem distribuída ao longo do ano analisado.

\subsection{NEE, Re e GPP}

O modelo de regressão não linear obteve resultado qualificado como "bom" para falhas de até $4 \mathrm{~h}$ com o coeficiente de determinação $\left(\mathrm{r}^{2}\right)$ entre $0.85<\mathrm{r}^{2} \leq 0.75$ e para falhas maiores de $4 \mathrm{~h}$ obteve a qualificação de "regular" $\left(0.5<\mathrm{r}^{2} \leq 0.75\right)$, conforme qualificação de Moffat et al., (2007). Os valores de $\mathrm{r}^{2}$ foram obtidos por meio da aplicação de falhas artificias de randomicamente distribuídas pela serie temporal de NEE e posteriormente preenchidos. Este processo foi repetido inúmeras vezes, comparando os valores preditos pela técnica com os observados.

Os valores de NEE durante o período de pousio são levemente superiores a zero, com valores médios diários durante o período próximos a $1 \mathrm{~g} \mathrm{C} \mathrm{m}^{-2} \mathrm{~d}^{-1}$. A grande diferença entre os valores de NEE ao longo do ano ocorre na época de cultivos de arroz destacados na Figura 2. Os valores de NEE obtidos durante a cultura de arroz tiveram um máximo de $-7,8 \mathrm{~g} \mathrm{C} \mathrm{m}^{-2} \mathrm{~d}^{-1}$, com Re máximo 


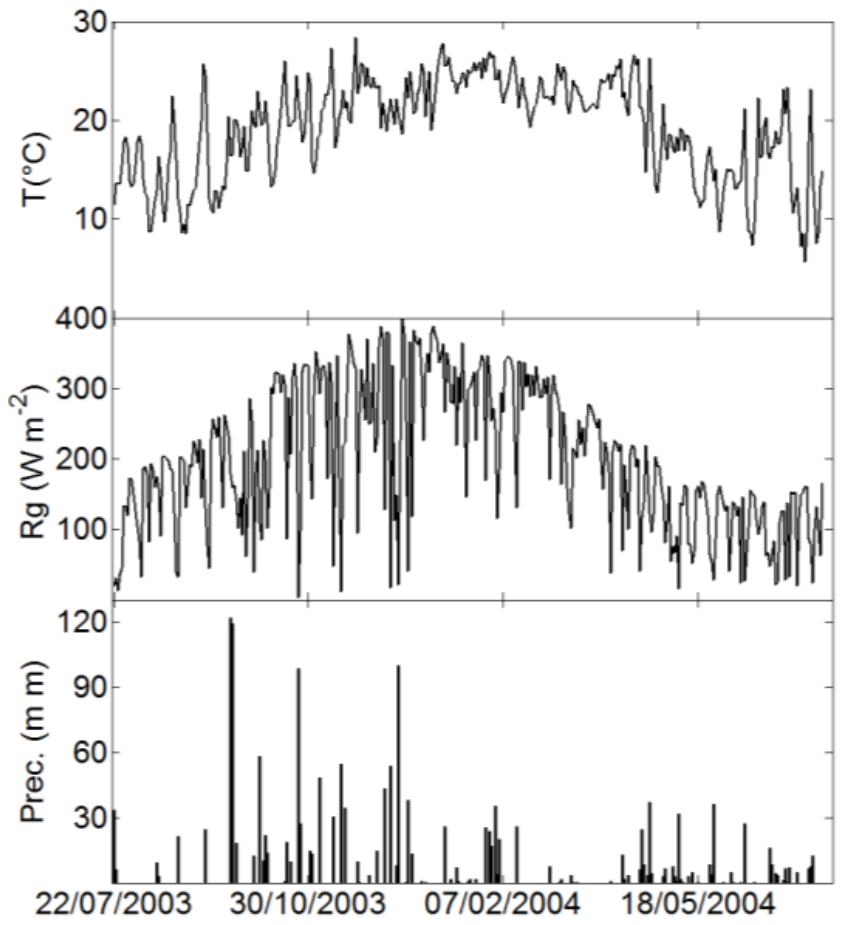

Figura 1- Médias diárias das variáveis meteorológicas para o sítio de Paraíso do Sul, entre 22/07/2003 e 21/07/2004: (a) Temperatura do ar, (b) Radiação global incidente. (c) Precipitação total diária.

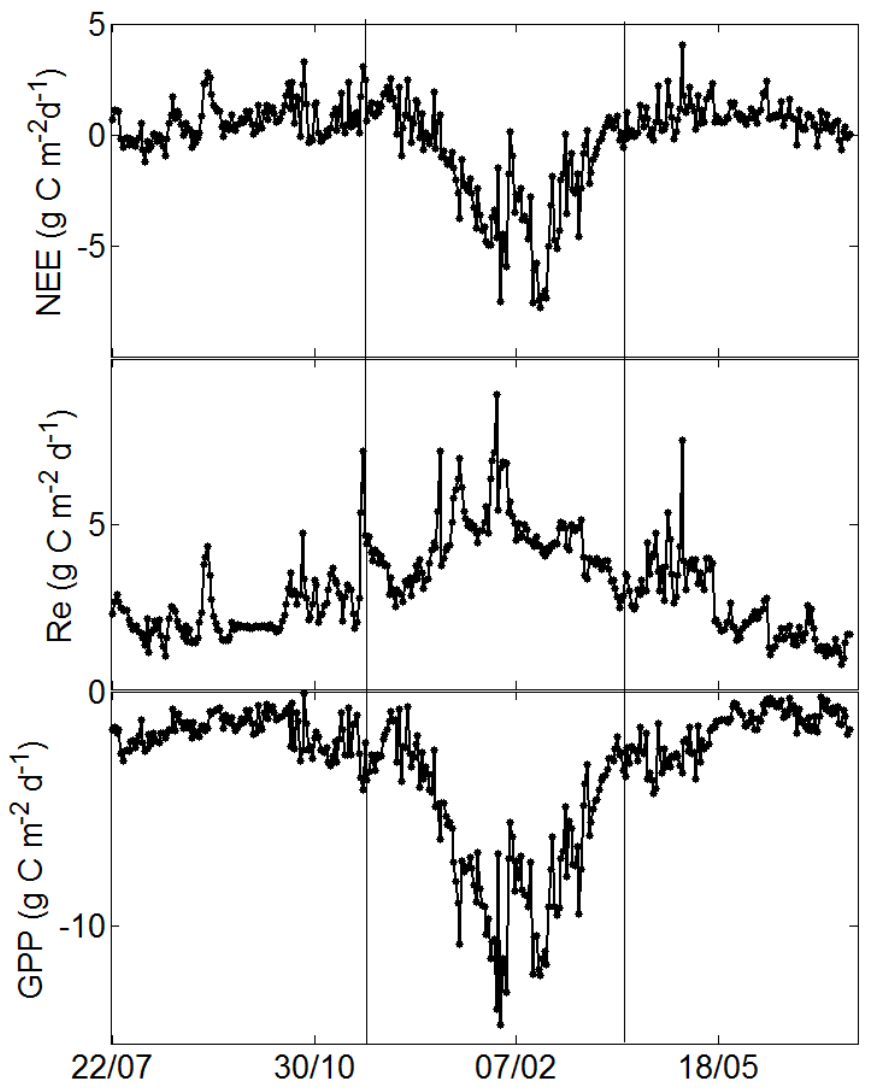

Figura 2- Valores médios diários de NEE, Re e GPP para Paraíso do Sul entre 22/07/2003 a 21/07/2004, com o período de cultivo de arroz destacado pelas linhas tracejadas entre 25/11/2003 a 04/04/2004. 
de $8,8 \mathrm{~g} \mathrm{C} \mathrm{m}^{-2} \mathrm{~d}^{-1}$ e GPP máximo de $-14,2 \mathrm{~g} \mathrm{C} \mathrm{m}^{-2} \mathrm{~d}^{-1}$.

Os valores acumulados de Re ao longo do período analisado foram de $1132 \mathrm{~g} \mathrm{C} \mathrm{m}^{-2}$ e o GPP de $-1175 \mathrm{~g} \mathrm{C}$ $\mathrm{m}^{-2}$. Para o período de cultivo de arroz o Re acumulado foi de $581 \mathrm{~g} \mathrm{C} \mathrm{m}^{-2}$ e o GPP de $-788 \mathrm{~g} \mathrm{C} \mathrm{m}^{-2}$.

$\mathrm{O}$ balanço final dos valores acumulados de NEE ao longo de todo o período foi de $-43 \mathrm{~g} \mathrm{C} \mathrm{m}^{-2}$ e no período de Cultivo de arroz foi de $-207 \mathrm{~g} \mathrm{C}^{-2}$.

\section{Conclusão}

O modelo de regressão não linear respondeu satisfatoriamente ao preencher as lacunas na série temporal de NEE. Os acumulados anuais de $\mathrm{CO}_{2}$ obtidos pela técnica de fechamento de falhas e partição de Carbono mostrou que a cultura de arroz irrigado no sul do Brasil funcionou como absorvedor de carbono de $207 \mathrm{~g} \mathrm{C} \mathrm{m}^{-2}$ no período de cultivo e, ao longo de um ano analisado (22/07/2003 a 21/07/2004), o agroecossistema funcionou como um absorvedor, acumulando $47 \mathrm{~g} \mathrm{C} \mathrm{m}^{-2}$ por ano.

\section{Referências}

AUBINET, M. et al. Estimates of the annual net carbon and water exchange of forests: The EUROFLUX methodology. Advances in Ecological Research, v. 30, p. 113-175, 2000.

AUBINET, M. Eddy covariance CO2-flux measurements in nocturnal conditions: An analysis of the problem. Ecological Applications, v.18, p.1368-1378, 2008

BALDOCCHI, D. et al. FLUXNET. A new tool to study the temporal and spatial variability of ecosystem-scale carbon dioxide, water vapor, and energy flux densities. Bulletin of the American Meteorological Society 82, 2415-2434, 2001.

Béziat P.; Ceschia, E., Dedieu, G. Carbon balance of a three crop succession over two cropland sites in South West France. Agricultural and Forest Meteorology, v.149, p.1628-1645, 2009.

FALGE, E. et al. Gap filling strategies for defensible annual sums of net ecosystem exchange.Agricultural and Forest Meteorology, v. 107, p. 43-69, 2001.

KAIMAL, J.; FINNIGAN, J. Atmospheric Boundary Layer Flows-Their Structure andMeasurement. New York: Oxford University Press, p.289, 1994

KIRSCHBAUM, M.U.F., EAMUS, D., GIFFORD, R.M. , Roxburgh, S.H., San,P.J, Definitionof some ecologicaltermscommonlyused in CarbonAccounting. NEE Workshop Proceedings: 18-20 April, 2001
LASSLOP, G., REICHSTEIN, M., PAPALE, D., RICHARDSON, A.D.; ARNETH, A.; BARR, A.; STOY, P.; WOHLFAHRT, G. Separation of net ecosystem exchange into assimilation and respiration using a light response curve approach: critical issues and global evaluation. Global Change Biology, v.16, p.187-208, 2010.

LLOYD, J.; TAYLOR, J.A. On the temperature dependence of soil respiration. Functional Ecology, v.8, p.315-323, 1994.

WEBB, E.; PEARMAN, G.; LEUNING, R. Correction of flux measurements for density effects due to heat and water vapor transfer. Quarterly Journal of the Royal Meteorological Society, v.106, p.85-100, 1980.

Moffat, A.M.; Papale, D.; Reichstein, M.; Barr, A.G.; Braswell, B.; Churkina, G.; Desai, A.R.; Falge, E.; Gove, J.H.; Heimann, M.; Hollinger, D.Y.; Hui, D.; Jarvis, A.J.; Kattge, J.; Noormets, A.; Richardson, A.D.; Stauch, V.J. Comprehensive comparison of gap filling techniques for net carbon fluxes. Agricultural and Forest Meteorology, v.147, p.209-232, 2007.

PAPALE, D. et al. Towards a standardized processing of Net EcosystemExchange measured with eddy covariance technique: algorithms and uncertainty estimation. Biogeosciences, v. 3, p 571-583, 2006

RUPPERT, J. et al. Innovative gap-filling strategy for annual sums of $\mathrm{CO} 2$ net ecosystem exchange. Agricultural and Forest Meteorology 138, p.5-18, 2006

ZEIGER, E., TAIZ, L. Fisiologia Vegetal, Porto Alegre: Armed , 4. ed., 200 